\title{
Microanatomy of the intestinal lymphatic system
}

\author{
Mark J. Miller ${ }^{a}$ and Rodney D. Newberry $b$ \\ aDepartment of Pathology and Immunology, Washington University School of Medicine, St. Louis, \\ Missouri \\ bepartment of Internal Medicine, Washington University School of Medicine, St. Louis, Missouri
}

\begin{abstract}
The intestinal lymphatic system is comprised of two non-communicating lymphatic networks; one containing the lacteals draining the villi and the connecting submucosal lymphatic network, and one containing the lymphatics that drain the intestine muscular layer. These systems deliver lymph into a common network of collecting lymphatics originating near the mesenteric border. The intestinal lymphatic system serves vital functions in the regulation of tissue fluid homeostasis, immune surveillance, and the transport of nutrients, and conversely this system is affected by, and directly contributes to, disease processes within the intestine. Recent discoveries of specific lymphatic markers, factors promoting lymphangiogenesis, and factors selectively affecting the development of intestinal lymphatics hold promise for unlocking the role of lymphatics in the pathogenesis of diseases affecting the intestine and for intestinal lymphatic selective therapies. Vital to progress in understanding how the intestinal lymphatic system functions is integrating of recent advances identifying molecular pathways for lymphatic growth and remodeling with advanced imaging modalities to observe lymphatic function and dysfunction in vivo.
\end{abstract}

\section{Keywords}

intestine; lymphatics; immunology; microscopy

\section{Introduction}

In higher vertebrates, two vascular systems, the blood vascular system and lymphatic system, serve vital and non-overlapping functions. The lymphatic system regulates tissue pressure and fluid status, provides a conduit for immune surveillance, and within the intestine, transports nutrients and other substances acquired from the intestinal lumen. While the lymphatic system was discovered nearly 400 years ago, the development and function of this system and how this system contributes to disease pathogenesis is still not well understood. Crucial to understanding the function of this second vascular system in the intestine is an understanding of the anatomy of the lymphatic system and how this system is affected by and contributes to disease. Here we will outline the microanatomy of the intestinal lymphatic system, highlight recent discoveries facilitating the study of lymphatic biology in the intestine, and discuss the potential of combining these advances with newer imaging techniques to investigate the role of lymphatics in disease pathogenesis.

Correspondence: Rodney D. Newberry, M.D., 660 S. Euclid Avenue Box 8124, St. Louis, MO 63110, rnewberry@im.wustl.edu, Mark J. Miller PhD, 660 S. Euclid Avenue Box 8118, St. Louis, MO 63110, miller@pathology.wustl.edu. 


\section{Results/Discussion}

\section{General organization of lymphatics system}

A large number of ultrastructural studies have revealed many details regarding the microanatomical arrangement of lymphatics in various tissues from multiple species (reviewed in Ohtani et al. ${ }^{1}$ ). While differences between organs and species have been observed, similarities between the lymphatic microanatomy from different organs and species can serve to suggest useful generalizations.

The lymphatic vasculature can largely be divided into two components, the initial lymphatic networks and the collecting lymphatic vessels. In general, the initial lymphatic networks contain blind-ended vessels that lack smooth muscle cells, are non-contractile, and lack valves. ${ }^{2}$ Initial lymphatic vessels can be attached to connective tissue within organs. ${ }^{3}$ Because initial lymphatics are non-contractile, they rely on other extrinsic forces, such as peristalsis, their connection to local connective tissue, and unique cell-cell junctions to fill and empty. Lymphatic endothelial cells in the initial lymphatic networks have overlapping flaps that serve a specialized function as unidirectional valves during the filling and emptying of the initial lymphatic network. ${ }^{4}, 5$ To form these flaps, the lymphatic endothelial cells in the initial lymphatics have vascular endothelial cadherin dependent button-like junctions positioned at the border of the flaps, thus anchoring the flaps and allowing the flaps to act as valves. ${ }^{6}$ The overlapping flaps allow spaces to open between lymphatic endothelial cells in the initial lymphatics during vessel expansion due to extrinsic forces to allow the lymphatic to fill with interstitial fluid. Conversely, these overlapping flaps allow these spaces to close during compression from extrinsic forces preventing lymph from returning to the interstitium during vessel emptying. It is currently unclear if the openings generated by these overlapping flaps contribute to leukocyte entry into the initial lymphatics.

Downstream of the initial lymphatics are the collecting lymphatics, which serve to transport lymph away from the initial lymphatics. Unlike the initial lymphatics, collecting lymphatic vessels contain smooth muscle and valves and are not attached to local connective tissue. ${ }^{1}$ Collecting lymphatics are comprised of a series of functional units termed lymphangions, which include a valve and a portion of the collecting lymphatic extending downstream to the next valve. ${ }^{7}$ The smooth muscle within the lymphangions allows for spontaneous contraction and, in conjunction with the valves, facilitates the transport of lymph to the adjacent downstream lymphangion away from the tissues.

\section{Anatomy of the Lymphatics System in the Small Intestine}

In the small intestine, the lymphatic system not only regulates tissue fluid homeostasis and promotes immune surveillance but also functions to transport lumenal substances including dietary fat and fat-soluble vitamins. Perhaps due to this additional role, the small intestine contains one of the more studied tissue lymphatic systems across many species. ${ }^{8-17}$

Because lymphatic specific markers were only recently identified, early studies relied upon microinjection of dyes and scanning electron microscopy of corrosion casts to investigate intestinal lymphatics. Despite these limitations, the findings from these studies support a general model of the microanatomy of intestinal lymphatics (Fig. 1) that appears to correlate with more recent immunohistochemical studies using lymphatic specific markers. ${ }^{1,} 14$

The intestine contains three layers of lymphatics, including (progressing from the lumenal surface to the serosal surface): the lacteals in the villi, a submucosal lymphatic network, and a lymphatic network in the smooth muscle layer. ${ }^{10}$ The number of lacteals in each villus varies depending upon the species and upon the region of the small intestine studied. ${ }^{2}, 14,15$ The lacteals connect with the submucosal lymphatic network at the base of the villi that have 
abundant interconnections. Injection of tracers into the submucosal network reveals that tracers are free to flow throughout the network and into adjacent villi, indicating that the lacteals and the submucosal network has few, if any valves. ${ }^{10}$ Most studies support that lacteals and the submucosal lymphatic network do not contain smooth muscle and consequently do not exhibit spontaneous contraction. However, studies of rat small intestine lymphatic corrosion casts using scanning electron microscopy revealed the presence of longitudinally oriented muscle cells associated with the lacteals. ${ }^{14}$ The inferred function of these cells is to constrict the lacteal and transfer lymph to the underlying submucosal lymphatic network. Injection of tracers into lacteals or the submucosal lymphatic network did not reveal a connection between these networks and the lymphatic network of the muscle layer. ${ }^{10}$ Likewise, injection of tracers into the lymphatic network of the muscle layer did not reveal communication with the lacteal or submucosal lymphatic network but did allow the tracer to freely move throughout the lymphatic network of the muscle layer. ${ }^{10}$ These observations suggest that the small intestine has two distinct lymphatic networks: one comprised of the lacteals and submucosal lymphatic network and one comprised of the lymphatic network of the muscle layer. Tracers move freely within each of these networks, suggesting that they contain few if any valves; however, tracers are not exchanged between the networks, indicating that there is no direct connection between the two networks. While the submucosal and muscle layer lymphatic networks have no direct connections, these two systems drain into common collecting lymphatics near the mesenteric border of the intestine. ${ }^{10}$ These collecting lymphatics contain valves and have spontaneous contractions to transport the lymph away from the intestinal segment.

While ultrasturctural studies have identified the lymphatic networks within the intestine, the pathways the lymph constituents take to reach the lacteals have been less well defined. Prelymphatic channels leading to initial lymphatics have been detected using tracers in various tissues including the liver, brain, and mesentery. ${ }^{18-20}$ Immunohistochemistry using a 5 '-nucleotidase to mark lymphatics failed to identify prelymphatic channels in the monkey mesentery and intestinal wall, indicating that these prelymphatic channels were not likely a direct component of the lymphatic network. ${ }^{9}$ Scanning electron micrograph studies of corrosion casts, in which the prelymphatic channels appear as non-endothelialized tissue channels, supports this implication. ${ }^{18}$ Using two-photon (2P) intravital microscopy, we have observed that intralumenally administered tracers fill tissue channels within the intestinal lamina propria that lead to a centrally located lacteal (see below). Thus, within the intestine prelymphatic tissue, channels provide a vital function delivering lymph constituents derived from both the blood vasculature and from lumenal substances.

\section{Intestinal lymphatics and disease}

Intestinal lymphatics are involved in a variety of disease processes ranging from congenital malformations resulting in primary lymphangeictasias to dynamic processes of lymphatic growth, remodeling, and inflammation. ${ }^{21,22}$ Inflammatory bowel diseases are chronic inflammatory conditions affecting the gastrointestinal tract. These diseases are largely divided into two groups that affect either all portions of the gastrointestinal tract (e.g., Crohn's disease) or the colon (e.g., ulcerative colitis). Several observations suggest that lymphatics may play a role in these diseases. Increased lymphatics are seen in ulcerative colitis; this correlation, along with the finding of lymphangiectasia, was particularly striking in areas with epithelial dysplasia. ${ }^{23}$ While lymphangiogenesis and lymphangiectasia were also seen in ileal and colonic Crohn's disease, ${ }^{24}$ other studies imply a more direct association between lymphatics and Crohn's disease pathogenesis. Lymphangitis can be a prominent component of infections and was one of the earliest noted pathologies in Crohn's disease. ${ }^{25-31}$ Furthermore, a model of Crohn's disease can be induced by sclerosis of regional intestinal lymphatics in rats and pigs. ${ }^{32,} 33$ These observations and the implied role 
of the microbiota in inflammatory bowel disease pathogenesis suggests that understanding the cause and consequences of lymphangitis could be a key to understanding Crohn's disease pathogenesis.

Recent advances identifying lymphatic specific markers and lymphatic specific growth factors have reinvigorated investigations in lymphatic biology. Multiple growth factors promoting lymphangiogenesis have been identified, including platelet derived growth factors, ${ }^{34}$ vascular endothelial growth factors (VEGF), ${ }^{35-38}$ insulin-like growth factors, ${ }^{39}$ and growth hormones. ${ }^{40}$ VEGFR-3 and its ligands VEGF-C and VEGF-D have been observed to play unique roles in promoting the development of new lymphatics in adults. ${ }^{41}$ Consequently, these growth factors may offer promise as therapies to promote or prevent lymphangiogenesis in intestinal diseases such as lymphangiectasias or inflammation. Furthermore, some VEGF family members may have selective roles in promoting physiologic or pathologic lymphangiogenesis during inflammation. In a murine model of chronic respiratory inflammation dendritic cells, macrophages, and neutrophils expressed VEGFR-3 ligands VEGFC and/or VEGFD and had beneficial effects on disease course. ${ }^{35} \mathrm{In}$ this model, VEGFC and VEGFD stimulated lymphangiogenesis through the VEGFR-3, and inhibition of VEGFR-3 worsened edema and disease. ${ }^{35}$ Conversely, in a murine model of cutaneous delayed type hypersensitivity, VEGF-A induced lymphangiogenesis appears to worsen inflammation due to the development of dysfunctional lymphatics. ${ }^{42}$ If these divergent roles for the VEGF family members remain true in other models and in other tissues, the selective targeting of VEGF family members holds significant promise as a therapy for inflammatory diseases.

While not highly investigated, there is precedent for factors to selectively affect the intestinal lymphatic system. Fasting-induced adipose factor (Fiaf) or angiopoietin-like protein 4 (Angpt14) is produced in the intestine and regulates the expression of prox 1 , a homeobox gene critical for lymphangiogenesis. ${ }^{43,}{ }^{44}$ Deletion of Fiaf resulted in a failure of blood and lymphatic vasculature partitioning in the small intestine. ${ }^{43}$ This defect was not observed in central or skin lymphatics, confirming the selectivity of Fiaf for development of the intestinal lymphatic system. ${ }^{43}$ These findings emphasize the uniqueness of the intestinal lymphatic system related to its role as a pathway for nutrient transport. Combined with the above observations, this holds promise for the potential to develop therapies selectively affecting intestinal lymphatic development and function during disease processes.

\section{Future modalities for studying the intestinal lymphatic system}

Although studies using fixed thin sections (EM and immunofluorescence) have provided fundamental information regarding the structure and function of the lymphatic network, these approaches have several limitations. First, when tissues are fixed and the interstitial pressure is lost, lymphatic vessels collapse and the native morphology is lost. Second, it is often difficult to interpolate a three-dimensional structure from two-dimensional data and precise structural data could be crucial for fully understanding the function of the various lymphatic networks in the gut. Finally, fixed sections provide only a "snap shot" in time and do not permit a direct analysis of cell dynamics during lymphatic flushing or leukocyte egress from peripheral tissue sites to secondary lymphoid organs.

Many of these limitations can be overcome with $2 \mathrm{P}$ microscopy. ${ }^{45}$ Two photon microscopy is high-resolution imaging technique that allows the spatiotemporal dynamics of individual cells to be studied in vivo. ${ }^{46,47}$ Two photon excitation occurs when two photons delivered by a near-infrared femtosecond pulsed laser (typically a Ti:Sapphire laser) are absorbed by a fluorophore to produce fluorescence emission. Because excitation occurs only at the point of focus, fluorescence emission is spatially constrained and photodamage in the sample is substantially reduced compared to single-photon techniques such as laser scanning confocal 
microscopy. The laser spot is then scanned through the specimen to build up optical sections (Z-stacks), which are acquired sequentially over time to generate three-dimensional timelapse images. Rendered multi-dimensional data sets are analyzed to determine cell velocity, shape, volume, number, intensity, and co-localization in three-dimensional space. Cells are commonly labeled for imaging by staining with fluorescent dyes (CellTracker, MolecularProbes) or by genetic engineering to express fluorescent proteins in the cell lineage of interest. In addition, $2 \mathrm{P}$ excitation can produce a second harmonic generation signal in many birefringent biological materials, including collagen in the skin and connective tissues. ${ }^{48,49,50}$ For example, excitation of collagen at $900 \mathrm{~nm}$ yields a second harmonic generation signal at $450 \mathrm{~nm}$ that provides a convenient tissue landmark in many tissues. In some cases, $2 \mathrm{P}$ excitation induces sufficient auto-fluorescence for individual cells to be visualized without dye-labeling, such as the case with macrophages (unpublished observations), skeletal muscle,${ }^{51}$ and pancreatic islets. ${ }^{52}$

Neurobiologists and developmental cell biologists were early adopters of $2 \mathrm{P}$ microscopy ${ }^{53,54}$ and more recently, immunologists have made extensive use of the technique to study single-cell dynamics during lymphocyte development, immunization, and infection in a variety of tissue types. ${ }^{55,56}$ The technique has significant potential for evaluating pathological changes in tissues associated with inflammatory diseases and cancer. ${ }^{57}$

Surprisingly, the application of $2 \mathrm{P}$ microscopy to study the gut is limited to only a handful of publications. Tutsch et al. used 2P microscopy to image calcium signals of individual $\mathrm{T}$ lymphocytes in the lamina propria of human intestine biopsies specimens ${ }^{58}$; in mice, $2 \mathrm{P}$ microscopy was used to acquire high-resolution three-dimensional images of fixed wholemount mouse intestine. ${ }^{59}$ Intravital $2 \mathrm{P}$ imaging of the mouse small intestine was first reported by Chieppa et al. and was used to study the intralumenal sampling behavior of lamina propria dendritic cells in real-time. ${ }^{60}$ Subsequently, intravital $2 \mathrm{P}$ microscopy of the duodenum was used to assess duodenocyte $\mathrm{pHi}$ in vivo using the $\mathrm{pH}$-sensitive dye SNARF-1F, ${ }^{61}$ while $2 \mathrm{P}$ intravital miscroscopy of the cecum was used to investigate DSSinduced damage to the cecal epithelium in actin-GFP transgenic mice. ${ }^{62} 2 \mathrm{P}$ microscopy was also to image the stomach ex vivo following Helicobacter pylori infection in mice ${ }^{63}$ and to image goblet cells in vivo as a marker of intestinal metaplasia of the stomach using an elegant $2 \mathrm{P}$ endoscopy approach. ${ }^{64}$

In terms of lymphatic network biology, the $2 \mathrm{P}$ technique has only rarely been used. In one study, the structure and mechanical properties of ex planted bovine lymphatic vessels was studied using $2 \mathrm{P}$ microscopy. ${ }^{65} \mathrm{In}$ a second study, $2 \mathrm{P}$ imaging was used to assess the realtime trafficking behavior of LP-DCs in surgically exposed lymphatic vessels in mice at the single-cell level. ${ }^{66}$

Building on the approach of Chieppa et al., ${ }^{60}$ our group has developed an intravital gut imaging preparation for mice that permits imaging from both serosal and luminal surfaces, while at the same time minimizing peristaltic movement (Fig. 2). This approach is poised to contribute greatly to lymphatic research because it permits cell dynamics and 3D tissue structure to be quantitatively analyzed in vivo. By exploiting the power of genetic approaches (i.e., transgenic and knockout mice), in vivo $2 \mathrm{P}$ imaging can provide insight into the molecular mechanisms that underlie leukocyte trafficking. For example, transgenic mice engineered to express fluorescent proteins in DCs, neutrophils, T cells, and other leukocyte populations would allow single-cell tracking of cells near lymphatic vessels and could be used to investigate how leukocytes locate and enter the lymphatics. Moreover, imaging mice deficient for specific adhesion molecules, chemokine receptors, or chemokines could help identify the key receptors and ligands that operate during lymphatic return. Real-time $2 \mathrm{P}$ 
imaging could provide a detailed picture of lymphatic endothelial cells that regulate the entry of soluble material and leukocytes. In addition to measuring the flow characteristics and cell trafficking in vivo, $2 \mathrm{P}$ microscopy could be used to document changes in the structure and morphology of the lymphatic network itself. In this regard, the generation of transgenic mice expressing fluorescent protein specifically in lymphatic endothelial cells is a high priority.

Beyond direct imaging of the structure of the intestinal lymphatic system, we envision $2 \mathrm{P}$ microscopy having a significant impact on the study of mucosal immune responses. Currently, we are using 2P microscopy to study the capture of antigen by DCs in the intestinal epithelium and submucosa. In the near future, we hope to use in vivo imaging to investigate the factors that regulate DC trafficking from the gut to draining lymphoid tissues, as well the spatiotemporal dynamics of antigen presentation during infection, tolerance, and autoimmunity. DC trafficking via lymphatic vessels serves an essential role during pathogenic infection by delivering foreign antigen to secondary lymphoid organs for antigen presentation and the induction of adaptive immune responses. However, the transport of food antigens and antigen from normal flora via the lymphatics is also likely to be an important mechanism for inducing and maintaining peripheral tolerance. If the cellular transport and presentation of antigen mechanisms is improperly regulated or aberrant lymphatic development or function occurs, these changes could contribute to the development of autoimmune and inflammatory diseases. Key to understanding the mechanisms of tolerance induction is to know when and where naïve, effector, and regulatory $\mathrm{T}$ cells recognize antigen. Previous reports have used changes in $\mathrm{T}$ cell dynamics as a robust indicator of antigen presentation in situ. Therefore, 2P time-lapse imaging of $\mathrm{T}$ cell dynamics could be instrumental for determining the anatomical location, kinetics, and relative strength of antigen stimulation under tolerogenic and immunogenic conditions.

\section{Conclusion}

The intestinal lymphatic system has several unique features, including its ability to transport lumenally absorbed nutrients, the presence of dual independent lymphatic networks, and the dependence upon intestine specific factors for its development. This lymphatic system is affected by and directly contributes to the pathogenesis of intestinal diseases. Combining state of the art in vivo imaging modalities with recent discoveries of the molecular pathways involved in lymphatic development, growth, and remodeling offer avenues to better understand the roles this unique lymphatic system plays in health and disease.

\section{Acknowledgments}

This work was supported in part by grants DK64798-RDN, AI083538-RDN and AI077600-MJM.

\section{References}

1. Ohtani O, Ohtani Y. Organization and developmental aspects of lymphatic vessels. Arch Histol Cytol. 2008; 71:1-22. [PubMed: 18622090]

2. Schmid-Schonbein GW. Microlymphatics and lymph flow. Physiol Rev. 1990; 70:987-1028. [PubMed: 2217560]

3. Leak LV, Burke JF. Ultrastructural Studies on the Lymphatic Anchoring Filaments. J Cell Biol. 1968; 36:129-149.

4. Mendoza E, Schmid-Schonbein GW. A model for mechanics of primary lymphatic valves. J Biomech Eng. 2003; 125:407-414. [PubMed: 12929246]

5. Trzewik J, et al. Evidence for a second valve system in lymphatics: endothelial microvalves. FASEB J. 2001; 15:1711-1717. [PubMed: 11481218] 
6. Baluk P, et al. Functionally specialized junctions between endothelial cells of lymphatic vessels. J Exp Med. 2007; 204:2349-2362. [PubMed: 17846148]

7. Mislin H. Active contractility of the lymphangion and coordination of lymphangion chains. Experientia. 1976; 32:820-822. [PubMed: 782900]

8. Azzali G. Structure, lymphatic vascularization and lymphocyte migration in mucosa-associated lymphoid tissue. Immunol Rev. 2003; 195:178-189. [PubMed: 12969318]

9. Kato S, Miura M, Miyauchi R. Structural organization of the initial lymphatics in the monkey mesentery and intestinal wall as revealed by an enzyme-histochemical method. Arch Histol Cytol. 1993; 56:149-160. [PubMed: 8373658]

10. Unthank JL, Bohlen HG. Lymphatic pathways and role of valves in lymph propulsion from small intestine. Am J Physiol. 1988; 254:G389-G398. [PubMed: 3348405]

11. Azzali G, Arcari ML. Ultrastructural and three dimensional aspects of the lymphatic vessels of the absorbing peripheral lymphatic apparatus in Peyer's patches of the rabbit. Anat Rec. 2000; 258:7179. [PubMed: 10603450]

12. Azzali G, Vitale M, Arcari ML. Ultrastructure of absorbing peripheral lymphatic vessel (ALPA) in guinea pig Peyer's patches. Microvasc Res. 2002; 64:289-301. [PubMed: 12204653]

13. Komuro T, Hashimot Y. Three-dimensional structure of the rat intestinal wall (mucosa and submucosa). Arch Histol Cytol. 1990; 53:1-21. [PubMed: 2194550]

14. Ohtani O. Three-dimensional organization of lymphatics and its relationship to blood vessels in rat small intestine. Cell Tissue Res. 1987; 248:365-374. [PubMed: 3581150]

15. Ohtani O, Ohtsuka A. Three-dimensional organization of lymphatics and their relationship to blood vessels in rabbit small intestine. A scanning electron microscopic study of corrosion casts. Arch Histol Jpn. 1985; 48:255-268. [PubMed: 4062499]

16. Yamanaka Y, Araki K, Ogata T. Three-dimensional organization of lymphatics in the dog small intestine: a scanning electron microscopic study on corrosion casts. Arch Histol Cytol. 1995; 58:465-474. [PubMed: 8562137]

17. Regoli M, et al. Arrangement of the small intestine lymphatic network in the Peyer's patches of the mouse. A light and transmission electron microscopic study. Ann Anat. 1995; 177:229-235. [PubMed: 7598220]

18. Casley-Smith JR, Foldi-Borsok E, Foldi M. The prelymphatic pathways of the brain as revealed by cervical lymphatic obstruction and the passage of particles. Br J Exp Pathol. 1976; 57:179-188. [PubMed: 773400]

19. Ohtani O, Ohtani Y. Lymph circulation in the liver. Anat Rec (Hoboken). 2008; 291:643-652. [PubMed: 18484610]

20. Castenholz A, Hauck G, Rettberg U. Light and electron microscopy of the structural organization of the tissue-lymphatic fluid drainage system in the mesentery: an experimental study. Lymphology. 1991; 24:82-92. [PubMed: 1921481]

21. Wen J, et al. Primary Intestinal Lymphangiectasia: Four Case Reports and a Review of the Literature. Dig Dis Sci.

22. Cueni LN, Detmar M. The lymphatic system in health and disease. Lymphat Res Biol. 2008; 6:109-122. [PubMed: 19093783]

23. Kaiserling E, Krober S, Geleff S. Lymphatic vessels in the colonic mucosa in ulcerative colitis. Lymphology. 2003; 36:52-61. [PubMed: 12926829]

24. Pedica F, et al. Lymphangiogenesis in Crohn's disease: an immunohistochemical study using monoclonal antibody D2-40. Virchows Arch. 2008; 452:57-63. [PubMed: 18040712]

25. Bonnetblanc JM, Bedane C. Erysipelas: recognition and management. Am J Clin Dermatol. 2003; 4:157-163. [PubMed: 12627991]

26. Costa LR, Spier SJ, Hirsh DC. Comparative molecular characterization of Corynebacterium pseudotuberculosis of different origin. Vet Microbiol. 1998; 62:135-143. [PubMed: 9695286]

27. Riet-Correa F, et al. Lechiguana (focal proliferative fibrogranulomatous panniculitis) in cattle. Vet Res Commun. 2000; 24:557-572. [PubMed: 11305747]

28. Kostman JR, DiNubile MJ. Nodular lymphangitis: a distinctive but often unrecognized syndrome. Ann Intern Med. 1993; 118:883-888. [PubMed: 8480962] 
29. Warren S, Sommers SC. Cicatrizing enteritis as a pathologic entity; analysis of 120 cases. Am J Pathol. 1948; 24:475-501. [PubMed: 18859355]

30. Meadows TR, Batsakis JG. Histopathological Spectrum of Regional Enteritis. Arch Surg. 1963; 87:976-982. [PubMed: 14063831]

31. Lockhart-Mummery HE, Morson BC. Crohn's Disease of the Large Intestine. Gut. 1964; 5:493509. [PubMed: 14244023]

32. Kalima TV. Experimental lymphatic obstruction in the ileum. Ann Chir Gynaecol Fenn. 1970; 59:187-201. [PubMed: 5491558]

33. Kalima TV, Saloniemi H, Rahko T. Experimental regional enteritis in pigs. Scand J Gastroenterol. 1976; 11:353-362. [PubMed: 935796]

34. Cao R, et al. PDGF-BB induces intratumoral lymphangiogenesis and promotes lymphatic metastasis. Cancer Cell. 2004; 6:333-345. [PubMed: 15488757]

35. Baluk P, et al. Pathogenesis of persistent lymphatic vessel hyperplasia in chronic airway inflammation. J Clin Invest. 2005; 115:247-257. [PubMed: 15668734]

36. Goldman J, et al. Cooperative and redundant roles of VEGFR-2 and VEGFR-3 signaling in adult lymphangiogenesis. FASEB J. 2007; 21:1003-1012. [PubMed: 17210781]

37. Roberts N, et al. Inhibition of VEGFR-3 activation with the antagonistic antibody more potently suppresses lymph node and distant metastases than inactivation of VEGFR-2. Cancer Res. 2006; 66:2650-2657. [PubMed: 16510584]

38. Bjorndahl MA, et al. Vascular endothelial growth factor-a promotes peritumoral lymphangiogenesis and lymphatic metastasis. Cancer Res. 2005; 65:9261-9268. [PubMed: 16230387]

39. Bjorndahl M, et al. Insulin-like growth factors 1 and 2 induce lymphangiogenesis in vivo. Proc Natl Acad Sci U S A. 2005; 102:15593-15598. [PubMed: 16230630]

40. Banziger-Tobler NE, et al. Growth hormone promotes lymphangiogenesis. Am J Pathol. 2008; 173:586-597. [PubMed: 18583315]

41. Pytowski B, et al. Complete and specific inhibition of adult lymphatic regeneration by a novel VEGFR-3 neutralizing antibody. J Natl Cancer Inst. 2005; 97:14-21. [PubMed: 15632376]

42. Kunstfeld R, et al. Induction of cutaneous delayed-type hypersensitivity reactions in VEGF-A transgenic mice results in chronic skin inflammation associated with persistent lymphatic hyperplasia. Blood. 2004; 104:1048-1057. [PubMed: 15100155]

43. Backhed F, et al. Postnatal lymphatic partitioning from the blood vasculature in the small intestine requires fasting-induced adipose factor. Proc Natl Acad Sci U S A. 2007; 104:606-611. [PubMed: 17202268]

44. Backhed F, et al. The gut microbiota as an environmental factor that regulates fat storage. Proc Natl Acad Sci U S A. 2004; 101:15718-15723. [PubMed: 15505215]

45. Denk W, Strickler JH, Webb WW. Two-photon laser scanning fluorescence microscopy. Science. 1990; 248:73-76. [PubMed: 2321027]

46. Cahalan MD, Parker I. Choreography of cell motility and interaction dynamics imaged by twophoton microscopy in lymphoid organs. Annu Rev Immunol. 2008; 26:585-626. [PubMed: 18173372]

47. Bousso P. T-cell activation by dendritic cells in the lymph node: lessons from the movies. Nat Rev Immunol. 2008

48. Gauderon R, Lukins PB, Sheppard CJ. Optimization of second-harmonic generation microscopy. Micron. 2001; 32:691-700. [PubMed: 11334739]

49. Zoumi A, Yeh A, Tromberg BJ. Imaging cells and extracellular matrix in vivo by using secondharmonic generation and two-photon excited fluorescence. Proc Natl Acad Sci U S A. 2002; 99:11014-11019. [PubMed: 12177437]

50. Friedl P, et al. Biological second and third harmonic generation microscopy. Curr Protoc Cell Biol. 2007:15. Chapter 4: Unit 4. [PubMed: 18228516]

51. Rothstein EC, et al. Skeletal muscle $\mathrm{NAD}(\mathrm{P}) \mathrm{H}$ two-photon fluorescence microscopy in vivo: topology and optical inner filters. Biophys J. 2005; 88:2165-2176. [PubMed: 15596503] 
52. Rocheleau JV, Piston DW. Two-photon excitation microscopy for the study of living cells and tissues. Curr Protoc Cell Biol. 2003:11. Chapter 4: Unit 4. [PubMed: 18228433]

53. So PT, et al. Two-photon excitation fluorescence microscopy. Annu Rev Biomed Eng. 2000; 2:399-429. [PubMed: 11701518]

54. Yuste R. Fluorescence microscopy today. Nat Methods. 2005; 2:902-904. [PubMed: 16299474]

55. Germain RN, et al. Dynamic imaging of the immune system: progress, pitfalls and promise. Nat Rev Immunol. 2006; 6:497-507. [PubMed: 16799470]

56. Konjufca V, Miller MJ. Two-photon microscopy of host-pathogen interactions: acquiring a dynamic picture of infection in vivo. Cell Microbiol. 2009; 11:551-559. [PubMed: 19170686]

57. Wang BG, Konig, Halbhuber KJ. Two-photon microscopy of deep intravital tissues and its merits in clinical research. J Microsc. 238:1-20. [PubMed: 20384833]

58. Tutsch E, et al. Two-photon analysis of calcium signals in T lymphocytes of intact lamina propria from human intestine. Eur J Immunol. 2004; 34:3477-3484. [PubMed: 15495161]

59. Appleton PL, et al. Preparation of wholemount mouse intestine for high-resolution threedimensional imaging using two-photon microscopy. J Microsc. 2009; 234:196-204. [PubMed: 19397748]

60. Chieppa M, et al. Dynamic imaging of dendritic cell extension into the small bowel lumen in response to epithelial cell TLR engagement. J Exp Med. 2006; 203:2841-2852. [PubMed: 17145958]

61. Sjoblom M, et al. Duodenal acidity "sensing" but not epithelial HCO3- supply is critically dependent on carbonic anhydrase II expression. Proc Natl Acad Sci U S A. 2009; 106:1309413099. [PubMed: 19622732]

62. Toiyama Y, et al. Intravital imaging of DSS-induced cecal mucosal damage in GFP-transgenic mice using two-photon microscopy. J Gastroenterol.

63. Kao JY, et al. Helicobacter pylori immune escape is mediated by dendritic cell-induced Treg skewing and Th17 suppression in mice. Gastroenterology. 138:1046-1054. [PubMed: 19931266]

64. Bao H, et al. Imaging of goblet cells as a marker for intestinal metaplasia of the stomach by onephoton and two-photon fluorescence endomicroscopy. J Biomed Opt. 2009; 14:064031. [PubMed: 20059269]

65. Arkill KP, Moger J, Winlove CP. The structure and mechanical properties of collecting lymphatic vessels: an investigation using multimodal nonlinear microscopy. J Anat.

66. Schulz O, et al. Intestinal CD103+, but not CX3CR1+, antigen sampling cells migrate in lymph and serve classical dendritic cell functions. J Exp Med. 2009; 206:3101-3114. [PubMed: 20008524]

67. Lindquist RL, et al. Visualizing dendritic cell networks in vivo. Nat Immunol. 2004; 5:1243-1250. [PubMed: 15543150] 


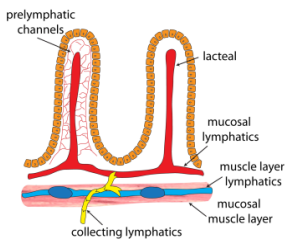

Figure 1.

The small intestine lymphatic system. Studies of the anatomy of intestinal lymphatics from multiple species have revealed that the small intestine contains two independent lymphatic networks that drain into a common network of collecting lymphatics. The lacteals, draining individual villi, connect with a network of lymphatics within the mucosal layer to form one distinct network (depicted in red), while the lymphatics of the mucosal muscle layer form a second distinct network (depicted in blue). Injection of tracers into these networks reveals free flow within each network but no exchange between networks, demonstrating the lack of valves within the networks and their independence. Both networks drain into contractile collecting lymphatics containing valves (depicted in yellow) located at the mesenteric border and largely outside of the intestine. Prelymphatic channels are non-endothelialized channels and therefore are not considered direct constituents of the lymphatic system. However, these prelymphatic channels play important roles in fluid homeostasis and, within the intestine, provide pathways to deliver lumenally absorbed substances to the lymphatic system. 


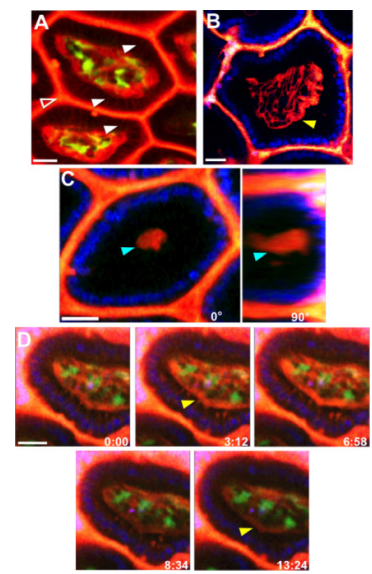

Figure 2.

Intravital 2P microscopy can image the intestinal lymphatic system in vivo in real time. A, Lamina propria dendritic cells (green) in a CD11c-YFP mouse ${ }^{67}$ injected intralumenally with $10 \mathrm{kD}$ dextran (red). Images are displayed as maximum intensity projections of 3D data sets. Unfilled white arrow shows luminal dextran staining between villi. Filled white arrows show examples of paracellular leak from the lumen into the lamina propria. Scale bar $=25$ $\mu \mathrm{m}$. B, Dextran-labeled prelymphatic vessels (yellow arrow) in a villus of a C57/BL6 mouse. DAPI (blue) was injected intralumenally to visualize the nuclei of intestinal epithelial cells. Scale bar $=25 \mu \mathrm{m}$. C, Left panel, dextran-labeled lacteal (red, cyan arrow) and epithelial cell nuclei (blue) in a villus. Right panel, orthogonal view projected at $90^{\circ}$. Scale bar $=20 \mu \mathrm{m}$. D, 2P time-lapse images of dextran (red) being flushed from the prelymphatic network. Clearing of dextran (yellow arrows) occurs over several minutes and is associated with peristalsis. CD11c-YFP ${ }^{+}$dendritic cells (green) in the lamina propria and epithelial cell nuclei blue. Time $=$ min:sec. Scale bar $=50 \mu \mathrm{m}$. 